This item was submitted to Loughborough's Research Repository by the author.

Items in Figshare are protected by copyright, with all rights reserved, unless otherwise indicated.

\title{
Magnetic short-range order in iron above Tc? Statistical mechanics with many-atom interactions
}

PLEASE CITE THE PUBLISHED VERSION

PUBLISHER

(C) Institute of Physics

LICENCE

CC BY-NC-ND 4.0

REPOSITORY RECORD

Chana, Kamaljeet S., J.H. Samson, M.U. Luchini, and V. Heine. 2019. "Magnetic Short-range Order in Iron Above Tc? Statistical Mechanics with Many-atom Interactions". figshare. https://hdl.handle.net/2134/2231. 


\title{
Magnetic short range order in iron above $T_{C}$ ?
}

\section{Statistical mechanics with many atom interactions}

K S Chana and J H Samson

Department of Physics, University of Technology, Loughborough, Leics LE11 3TU, UK

M U Luchini

Theoretische Physik, ETH-Hönggerberg, CH-8093, Zürich, Switzerland

Volker Heine

Cavendish Laboratory, Madingley Road, Cambridge CB3 0HE, UK

Short title: Short range order in Fe above $T_{C}$ ?

PACS. 75.10L - Band and itinerant models

PACS. 75.50B - Ferromagnetism of Fe and its alloys

\begin{abstract}
An effective spin Hamiltonian for iron has been derived previously from electronic structure calculations; there are strong many-atom interactions. The exchange interactions $J_{i j}$ depend on the surrounding magnetic order. We investigate the magnetisation and spin correlation function in iron on this basis in two ways: by fitting the $J_{i j}$ to an analytically soluble Hamiltonian (an extended spherical model) and by Monte Carlo simulation. Short-range manyatom interactions do not lead to substantial short range order in the paramagnetic state; the behaviour closely resembles that of the nearest-neighbour Heisenberg model. The main effect is a slight distortion of the magnetisation curve below $T_{\mathrm{C}}$. Longer range oscillatory pair interactions are needed for short-range order, but are not supported by the electronic structure data. We conclude that calculations of the electronic energy in static configurations do not lead to unusual short range order.
\end{abstract}

Published in J Phys: Conden. Matter 3 6455-6471 (1991) 


\section{Introduction}

Considerable controversy has surrounded the paramagnetic phase of metallic magnets. It is now generally accepted that the ground state can be well described by band theory and the Stoner model, and the phase transition by disordering of magnetic moments. An unresolved issue is that of the existence and extent of magnetic short range order (SRO) above the Curie temperature $T_{\mathrm{C}}$, in excess of that normally expected as critical fluctuations in a short-range Heisenberg model. The controversy was started by the inelastic neutron scattering data of Mook et al (1973), on nickel, and Lynn (1975), on iron. Their observations indicated the persistence of spin waves well above $T_{\mathrm{C}}$. Shirane (1984) and Shirane et al (1986) however interpreted the constant energy peaks in $\mathrm{Fe}$ as slices of a simple paramagnetic scattering function without the need to invoke substantial SRO or propagating spin waves. The main distinction between $\mathrm{Fe}$ and a typical magnetic insulator is that considerably less high $\mathbf{q}$ scattering is observed in the case of $\mathrm{Fe}$, and the integrated structure factor is less than the expected $S(S+1)$ (Brown et al 1982, Johnson et al 1987). Two schools of thought emerged from these observations. On the one hand the fluctuating local band theory (FLBT) of Korenman et al (1977) assumed SRO to exist from the outset; above $T_{\mathrm{C}}$ the spin direction varies sufficiently smoothly for a local band structure and broadened spin waves to be supported. On the other hand Edwards $(1980,1983)$ argued that this interpretation was incompatible with thermal and susceptibility data which showed a normal transition, and that SRO was not needed to explain the neutron data, which cover a restricted energy window. The disordered local moment (DLM) picture of Hubbard (1979), Hasegawa (1980) and others, in which the nearest-neighbour correlation is zero in the paramagnetic state, is compatible with the susceptibility and thermal data. Lowde et al (1983) describe a further model of an itinerant magnet with coarse-grained disorder: large blocks of reversed magnetisation develop at temperatures just below $T_{\mathrm{C}}$. These are larger than those normally expected from critical fluctuations. This is an alternative picture to the smooth magnetisation of FLBT. They interpret their neutron data on an impure sample of nickel in terms of such a block model. Angle-resolved photoemission studies of the band splitting in the electronic structure of paramagnetic iron also suggest the existence of SRO (Haines et al 1985, Kisker et al 1985).

The picture was then one of some confusion as to the high-temperature state of an itinerant magnet. Can we make do with a DLM description as is appropriate for an insulator such as EuO? Or is there substantial SRO and, if so, is it of the smooth FLBT type or in the form of blocks? If the transition were driven entirely by spin waves, then the wave vector at which disorder occurs should be related to the maximum wave vector $q_{\mathrm{c}}$ of spin waves; this corresponds to the zone boundary for insulators, but to the point at which the spin waves enter the Stoner continuum for itinerant magnets (Heine and Joynt 1988). Perhaps there was a direct way of finding the dominant configurations of the magnetisation through electronic structure calculations of the energies of some trial configurations: if the energies of certain configurations are substantially lower than would be expected from a Heisenberg model, they would contribute more to the thermodynamic ensemble. Now the recursion method (see e.g. Haydock 1980) provides a method for calculating electronic properties of non-periodic systems, such as the energies of random configurations of the magnetisation. These ideas led to our programme of work on iron (Lin-Chung and Holden, Heine et al 1981, You and Heine, Holden and You 1982, Samson 1983, Small and Heine 1984, Luchini and Heine 1989, 1991), mostly concerned with the energetics of configurations of the magnetisation rather than the statistical mechanics. We calculate the energy of the electron gas in an exchange field $\left\{\boldsymbol{\Delta}_{i}\right\}$, an effective magnetic field on each site; this will give us an effective spin Hamiltonian.

The overall picture, that the statistical mechanics of an itinerant magnet should be that of a classical spin Hamiltonian of this form, is based on the Hubbard model, which we take to be a microscopic description of Fe. A spin Hamiltonian emerges from the Hubbard model as a static approximation to a functional integral (see e.g. Hubbard 1979); the statistical mechanics of an itinerant magnet is that of a classical spin 
Hamiltonian given by the energy of non-interacting electrons in an exchange field. The band parameters and exchange integrals are obtained from fits to paramagnetic and ferromagnetic band structures. Our main approximation is then the static approximation.

There are various ways in which the resulting data on the energetics can be used. One can attempt to fit the data to a classical Heisenberg Hamiltonian of the form

$$
H=-\sum_{l j} J_{i j} \hat{\mathbf{e}}_{i} \cdot \hat{\mathbf{e}}_{j}
$$

where $\hat{\mathbf{e}}_{i}$ is the direction of the magnetic moment on atom $i$. The magnetic moment on atom $i$ is $m \hat{\mathbf{e}}_{i}$ and the exchange splitting is $\Delta \hat{\mathbf{e}}_{i}$; we have absorbed the spin into the $J_{i j}$. This assumes pairwise exchange interactions $J_{i j}$ (which could be of fairly long range). Alternatively, one can calculate the energies $H\left(\left\{\hat{\mathbf{e}}_{i}\right\}\right)$ of a number of configurations and define the exchange interactions as

$$
J_{i j}^{\mu v}=-2\left(\frac{\partial^{2} H}{\partial \hat{e}_{i}^{\mu} \partial \hat{e}_{j}^{v}}\right) \text {, where } \mu, v=x, y \text {, or } z \text {. }
$$

These are in general functions of the exchange field; they correspond to the $J_{i j}$ of equation (1.1) only if they are constant and isotropic in spin space. We will take equation (1.2) as our definition of $J_{i j}$. The dominant fluctuations (in iron at least) are assumed to be transverse; the magnitude of the magnetic moment in iron is found to vary little between the fully aligned ferromagnetic (FM) and DLM states (Samson 1983), suggesting that although the Heisenberg Hamiltonian may not be appropriate, the phase space is the same.

A further approach, one that assumes less about the form of the Hamiltonian, is to calculate the energies of various configurations and attach an entropy to each. The entropy corresponds to the number of configurations in phase space which are "similar to" to the chosen configuration, for example by finding the equivalent frozen spin wave

$$
\boldsymbol{\Delta}_{i}=\Delta\left(\sin \theta \cos \mathbf{q} \cdot \mathbf{r}_{i}, \sin \theta \sin \mathbf{q} \cdot \mathbf{r}_{i}, \cos \theta\right)
$$

There are three parameters here: the amplitude $\Delta$ of the exchange field, the order parameter or reduced magnetisation $(\cos \theta)$ and a SRO parameter, the spin wave wave vector $\mathbf{q}$. The thermodynamics can then be found by minimising the free energy:

$$
F=\min _{\Delta, \theta, \mathbf{q}}[E(\Delta, \theta, \mathbf{q})-T S(\Delta, \theta, \mathbf{q})]
$$

The entropy may be calculated in closed form in terms of lattice Green functions. Such an idea was proposed by Heine and Joynt (1988) and developed by Samson (1989). This reduces to mean field theory (MFT) if the energy is independent of the SRO parameter, and to the spherical model of Berlin and Kac (1952) if the energy is linear in the order parameter and the SRO parameter. We will therefore call this approach the extended spherical model (ESM).

Suppose we only know the dependence of the energy and entropy on one parameter, which we shall call the order; we have deliberately left this vaguely defined for the present. It is some quantity that falls from 1 in the FM state to 0 in the DLM state and depends on both the magnetisation and the SRO. Suppose also that there is a strong kink in the energy as a function of order, as described by Heine and Joynt (1988) 
and Luchini and Heine (1991a,b) (referred to below as LH), so that the energy rises at first slowly and then much more rapidly with decreasing order. This will indeed tend to favour states with that value of the order. But is this long- or short-range order? Let us consider all microstates consistent with that value of the order in the thermodynamic limit. If the order is large, almost all of these microstates will have non-zero magnetisation. If the order is small, almost all of these microstates will have zero magnetisation (strictly $\mathrm{O}(1 / \sqrt{ } N)$ ). The value of the order at which the magnetisation becomes non-zero will be the SRO at $T_{\mathrm{C}}$. This means that the kink in the energies will either lead to a distorted magnetisation curve or to substantial SRO above $T_{\mathrm{C}}$, depending on whether the kink comes above or below the critical value of the order. If the order is a weighted sum of terms such as $\left\langle\boldsymbol{\Delta}_{i} . \boldsymbol{\Delta}_{j}\right\rangle$, its critical value may be calculated by the ESM (Samson 1989); for example, if the order is taken to be the nearest-neighbour correlation in a BCC lattice, the critical value is 0.282. If the order depends on longer-range correlations, the critical value of order may be higher (and the critical temperature correspondingly lower). We must therefore choose a definition of the order carefully, so that the surfaces of constant order approximate constant energy surfaces in phase space.

Heine and Joynt (1988) argue that the magnon cutoff $q_{\mathrm{c}}$ should show up as a kink in a plot of the energies of configurations against the wave vector $q$ of the disorder as the magnons enter the Stoner continuum. A pronounced kink at some length scale might determine the amount of order; the paramagnetic state would be broken up into domains of this size. Such a kink was indeed seen in the first d-band tightbinding calculations of spin spirals, configurations of the form (1.3) with $\theta=\pi / 2$ (You and Heine 1982, Holden and You 1982). They also studied alternating tilt configurations, corresponding to zone boundary spin waves ( $\sin \mathbf{q} \cdot \mathbf{r}_{i}= \pm 1$ in equation (1.3)). Luchini and Heine (1989) extended these calculations by using a full spd tight-binding Hamiltonian, finding only a small kink at $q_{\mathrm{c}}$. Fitting these energies to a Heisenberg model leads to values for the exchange interactions $J_{i j}$ and hence the Curie temperature and spin wave stiffness $D$. These interactions are of fairly long range and oscillate in sign. A study of these energies by the ESM has however failed to find any support for the existence of SRO (Samson 1989). It is difficult to see how SRO can be obtained from pure pair interactions of the form (1.1) without contrived coefficients which the spin spiral data do not support (Shastry et al 1981).

Lin-Chung and Holden (1981) however found substantial many-atom interactions, calling into question any such fitting to a Heisenberg model. They extracted exchange interactions from energy differences, but these are likely to be inaccurate because of the difficulty of calculating the total energy of a configuration. The exchange interactions are effectively the second derivative of the Hamiltonian, and it would be of use to find these more directly. Small and Heine (1984) developed a method for calculating the exchange interactions in terms of the "couple" exerted by one spin on a neighbour, or the transferred moment at one site due to an exchange splitting on another - in effect the first derivative of the Hamiltonian. LH have shown that the exchange interactions as defined as in equation (1.2) are strongly affected by the amount of order in the surrounding shells. Since it is these results that form the basis of the present work, we now go into them in more detail.

LH set up random configurations in Fe with given average magnetisation

$$
<\hat{\mathbf{e}}_{i}>=(0,0, \cos \theta)
$$

and determined the effective nearest-neighbour and next-nearest-neighbour exchange interactions $J_{1}$ and $J_{2}$ as a function of $\theta$ by the couple method, in a way which gives them as second derivatives of the Hamiltonian as defined in equation (1.2). In the FM state $(\theta=0) J_{1}$ is slightly greater than $J_{2}$. As the system disorders with increasing temperature, $J_{1}$ increases and $J_{2}$ decreases (figure 1 ), so that at the DLM end the interaction is almost entirely between nearest neighbours. Longer range interactions are smaller in magnitude and fall 
off rapidly with increasing disorder. An independent check has been made of the sum $J_{\text {tot }}$ of the $J_{i j}$; this agrees with the values used for $J_{1}$ and $J_{2}$, so that longer range interactions are unimportant. We are assuming these interactions to be isotropic. From symmetry considerations, the $J_{i j}$ must be even functions of magnetisation; we therefore fit them to the form

$$
J_{i j}=A_{i j}+B_{i j}[\sigma(i, j)]^{2}
$$

Here $\sigma$ is the average magnetisation on shells of atoms "near" $i$ and $j$. (The meaning of "near" is discussed in $\S 2$.) The values of $A$ and $B$ for nearest and next-nearest neighbours are

$$
\left.\begin{array}{l}
A_{1}=2.5 \mathrm{mRy} \\
B_{1}=-1.5 \mathrm{mRy} \\
A_{2}=0.2 \mathrm{mRy} \\
B_{2}=0.6 \mathrm{mRy}
\end{array}\right\} .
$$

The variation is due in some way to the change in the band structure on disordering. LH argue that the range of exchange interactions is reduced in the disordered state as a result of the reduction in electron mean free path through disorder scattering. Heine et al (1990) show how the behaviour of the couplings may also be understood through perturbation theory. Hubbard (1979) had also observed the effective exchange interaction to increase with disorder. The tendency for the interactions to be of short range in the DLM state was also noted by Oguchi et al (1983), whose CPA calculations show that the nearest neighbour interaction

dominates. Lipiński (1989) does however find large second- and third-neighbour interactions in the DLM state, also in the CPA. The Hamiltonian is in any case rather unusual and we shall be investigating its consequences. This behaviour of the exchange interactions effectively means that magnetic excitations have low energy in the FM state. As the disorder increases, the system becomes stiffer towards further disorder, leading LH to speculate that substantial SRO remains in the paramagnetic state.

We investigate this idea both analytically, by the ESM, and numerically, by Monte Carlo simulation, comparing the above model of Fe with a classical Heisenberg ferromagnet. We look at the spin correlation function in particular, in order to investigate the degree of SRO in the paramagnetic phase and the existence or otherwise of clusters in the ferromagnetic phase. If the correlation for nearest and next-nearest neighbours is greater than in the corresponding Heisenberg model, we can conclude that substantial SRO exists above $T_{\mathrm{C}}$. We must say now that our calculations show no evidence of substantial SRO, and conclude that static electronic structure calculations do not lead to unusual amounts of SRO. This is in agreement with the heat capacity and susceptibility data, but does not describe the neutron data, which essentially give a timeaveraged correlation function; we do not claim to have a Hamiltonian for the dynamics.

In the next section we discuss the Hamiltonians we shall be using and the type of Hamiltonian that would lead to SRO. It appears to be difficult to find any Hamiltonian with short-range many-atom interactions that would lead to SRO. The exchange interactions for iron are fitted to a Hamiltonian that can be solved exactly in the ESM, and the results so obtained are described in $\S 3$. The magnetisation is affected by the many-atom interactions, but the SRO is unaffected. The ESM ignores higher-order correlations that may be important in this case; for this reason, we have carried out Monte Carlo simulations, as reported in $\S 4$. This shows the same trends. Our results for the absence of SRO seem to be sufficiently robust that our 
conclusions will not be affected by the details of the fitting, as shown by our results for a model with exaggerated non-pairwise interactions. We conclude, in $\S 5$, that the data for iron do not support the existence of substantial SRO.

\section{Hamiltonian}

We shall be looking at several Hamiltonians, taking as a reference the classical nearest-neighbour Heisenberg model (nnHm) in equation (1.1). The effective spin Hamiltonian relevant for iron is an immensely complicated functional of the magnetisation, even in the static approximation. To study its statistical mechanics, whether analytically or by simulation, requires extrapolation from the small subset of phase space for which the energies have been calculated, which inevitably leads to some ambiguities.

The basic idea is that the exchange interactions in iron are stronger in the DLM state than in the FM state, as shown in equations (1.6-7). $J_{i j}$ depends on the band structure and therefore on the order in the surrounding shell through the parameter

$$
\sigma(i, j)=\left|\frac{\sum_{k \in S(i, j)} \hat{\mathbf{e}}_{k}}{z(i, j)}\right|,
$$

where $S(i, j)$ is the shell of neighbours of atoms $i$ and $j$, and $z(i, j)$ is the number of atoms in that shell. The question now arises of the degree of locality: how large a shell $S(i, j)$ is needed to define the interactions? One limit is for $S(i, j)$ to encompass the entire solid. This then gives us a type of MFT, where the (shortrange) interactions depend on the (long-range) order parameter. Let us consider a nearest-neighbour interaction $J$. Suppose the zero-field magnetisation in the $n n H m$ to be a function $M_{\mathrm{H}}\left(k_{\mathrm{B}} T / J\right)$; the magnetisation in the non-pairwise model will then be

$$
M(T)=M_{\mathrm{H}}\left(k_{\mathrm{B}} T /\left(A+B M^{2}\right)\right)
$$

to be solved self-consistently. If $J$ increases with decreasing magnetisation, the magnetisation will initially fall more rapidly with reduced temperature $T / T_{\mathrm{C}}$ than in a Heisenberg model. The magnetisation curves will be distorted below $T_{\mathrm{C}}$. All zero-field quantities above $T_{\mathrm{C}}$ will be identical to those in the Heisenberg model. If the shell $S(i, j)$ is fairly large, equation (2.2) still provides a good "mean field" solution of the problem that reduces to the Heisenberg model in the case $B=0$. The other limit is for $S(i, j)$ to consist of only the atoms $i$ and $j$ themselves. This leads to a Hamiltonian with biquadratic pair interactions, as studied by Brown (1971) and others. The larger the shell, the smaller the fluctuations in $J$ and the smaller the deviation from the Heisenberg model. LH find that the interactions depend principally on the nearest-neighbour shell. On that basis, we will take the shell to consist of the nearest neighbours of $i$ and $j$. For $J_{1}$, this consists of 16 sites (including $i$ and $j$ ); for $J_{2}$, this consists of 12 sites.

It must be stressed that the exchange parameters calculated by $\mathrm{LH}$ are the second derivative of the Hamiltonian with respect to the directions of the magnetisation, as in equation (1.2), and not the coefficients in a Hamiltonian of the form (1.1). To demonstrate the importance of this distinction, we first consider the extreme case $B_{1}=-A_{1}$ or 
in which the exchange interaction vanishes in the FM state. Suppose that this is the coefficient in a Hamiltonian of the form (1.1). The energy of the ferromagnetically ordered state is zero, as $J_{1}=0$; however, it is unstable towards the reversal of a spin. Reversing a spin turns on ferromagnetic interactions in the neighbourhood of the spin, the number of bonds depending on the degree of locality of the Hamiltonian. Since the majority of these bonds will be formed between ferromagnetically aligned spins, the total energy will be reduced, and the ferromagnetic state will be unstable. Similarly, the energy of a domain wall will be negative. Now if $J_{1}(\theta=0) / J_{1}(\theta=\pi / 2)$ is just large enough to stabilise the FM state, one could imagine that the domain wall energies will be small and the reversed-spin clusters described by Lowde et al (1983) would be observed at finite temperatures. Our Hamiltonian is however not of this form. The calculations of LH give the second derivative of the Hamiltonian; any instability of the FM state would show up in these energies.

We do not have a simple expression for the Hamiltonian itself, but only for its second derivative $J_{i j}$. We have attempted to integrate this twice to obtain the Hamiltonian: the quadratic form of the $J_{i j}$ in equation (1.6) suggests a quartic Hamiltonian with an inconveniently large number of 4-spin terms. We have tried to fit the exchange interactions to four-site coefficients arising from a fourth order perturbation expansion of the electronic energy in powers of the exchange field, but no reasonable fit was obtained, in the sense that the coefficients did not fall off in the expected way with the size of the four-site loops (unpublished). This may be due to the existence of higher order terms, or to the localisation effect mentioned above.

Although we have not succeeded in deriving a complete Hamiltonian, there are two ways in which we can use the $J_{i j}$. Firstly, one can start with the ferromagnetic state and rotate all the spins by the same angle $\theta$ in random directions away from the $z$ axis. The interactions can then be integrated, as shown in section 3 , to give the dependence of the energy on order. This is

$$
V\left(\sigma^{2}\right)=V(0)-A \sigma^{2}-\frac{1}{2} B \sigma^{4}
$$

Secondly, $\mathrm{LH}$ found that the couple acting on atom $i$ as it is rotated in a random environment is nearly proportional to $\sin \theta_{i}$ over the full range of $\theta_{i}$ from 0 to $\pi$, where $\theta_{i}$ is the angle with respect to the direction of magnetisation. One can then use the parameters in equations (1.6-7) to give the change in the energy on rotating a single spin in an arbitrary environment. This is precisely the information required for a Monte Carlo simulation. The difficulty of not having an explicit Hamiltonian does not therefore vitiate a Monte Carlo calculation. Section 4 gives the results of such a calculation.

\section{Our calculations will focus principally on four systems:}

I the nearest-neighbour Heisenberg model ( $\mathrm{nnHm})$ in equation (1.1).

II iron with many-atom interactions, as parametrised by equations (1.6-7).

III iron with pairwise exchange interactions after Luchini and Heine (1989). Two fits were used: (1) $J_{1}=0.68 \mathrm{mRy}, J_{2}=1.67 \mathrm{mRy}, J_{3}=-0.62 \mathrm{mRy}, J_{4}=0.17 \mathrm{mRy}, J_{5} \ldots=0$ and (2) $J_{1}=1.34 \mathrm{mRy}, J_{2}=0.73 \mathrm{mRy}, J_{3}=-$ $0.36 \mathrm{mRy}, J_{4}=0.05 \mathrm{mRy}, J_{5} \ldots=0$.

IV the extreme case of equation (2.3), in which the exchange interaction vanishes in the FM state.

The exchange interactions referred to here are as defined in equation (1.2). We have investigated these systems by the ESM and (with the exception of III) Monte Carlo simulation. 


\section{The Extended Spherical Model (ESM)}

This is an approximation for the statistical mechanics of a Hamiltonian whose functional form is not known but whose values are known at selected points in phase space (Samson 1989). It involves splitting the problem into an energy $E$ and an entropy $S$. The energy is the value of the Hamiltonian for the configuration in question; the values used here have been calculated by the recursion method. The entropy is the logarithm of that area of phase space which we assume to be degenerate with the chosen configuration. In practice we approximate the Hamiltonian $H\left(\left\{\boldsymbol{\Delta}_{i}\right\}\right)$ by an arbitrary function $V_{\mathrm{T}}(\Delta, \theta, c)$ of three macroscopic quantities: the amplitude $\Delta$ of the exchange field, the order parameter $\Delta \cos \theta$ and a $S R O$ parameter $c$ :

$$
\begin{aligned}
& \Delta^{2}=\frac{1}{N} \sum_{i=1}^{N} \Delta_{i}^{2} \\
& \Delta \cos \theta=\left|\frac{1}{N} \sum_{i=1}^{N} \boldsymbol{\Delta}_{i}\right| \\
& \left(\Delta^{2} \sin ^{2} \theta\right) c=\frac{1}{N} \sum_{i, j=1}^{N} \lambda_{i j} \boldsymbol{\Delta}_{i} \cdot \Delta_{j}-\left(\frac{1}{N} \sum_{i=1}^{N} \boldsymbol{\Delta}_{i}\right)^{2} \\
& \sum_{i, j=1}^{N} \lambda_{i j}=1,
\end{aligned}
$$

where $N$ is the number of atoms and $\left\{\lambda_{i j}\right\}$ are coefficients, describing the range of the correlations, that may be fitted to the Hamiltonian. If $\lambda_{i j}=1 / z$ for nearest neighbours and zero otherwise, $z$ being the coordination number, $c$ becomes the nearest-neighbour correlation. The coefficients $\left\{\lambda_{i j}\right\}$ could depend on the other parameters. The entropy $S(\Delta, \theta, c)$ is the logarithm of the area of phase space subject to the three constraints (3.1-3) and can be found in closed form in terms of lattice Green functions. The free energy $V_{\mathrm{T}}-T S$ is then minimised with respect to the parameters. Samson (1989) has applied this approach to the spin spiral data of Luchini and Heine (1989). He did not take account of their alternating tilt data, as those configurations refer to states with ferromagnetic order. In the case where $c$ is the nearest neighbour correlation, its value at $T_{\mathrm{C}}$ is small ( 0.282 for a BCC lattice); to obtain SRO one would require the Hamiltonian to depend on a longerrange set of $\lambda_{i j}$. In that case however, a fit of the [100] spin spirals to longer-range pairwise interactions shows very little difference from a fit to nearest-neighbour anharmonic interactions. The pairwise exchange interactions in system III are those calculated from a fit to both spin spiral and alternating tilt configurations (Luchini and Heine 1989) and will lead to a slightly enhanced SRO.

For pairwise interactions the ESM reduces to the spherical model of Berlin and Kac (1952). The fitting procedure we use for many-atom interactions is to substitute the Hamiltonian in the form $V_{\mathrm{T}}(\Delta, \theta, c)$ into equation (1.2) and compare the resulting $J_{i j}$ with equation (1.6). To obtain a closed set of equations, we need to fit the mean square magnetisation $\sigma^{2}$ of the shell $S(i, j)$ of neighbours of atoms $i$ and $j$, to a function of the parameters. This is 
$[\sigma(i, j)]^{2}=\cos ^{2} \theta+c^{\prime} \sin ^{2} \theta$

where $c^{\prime}=\frac{1}{z(i, j)^{2}} \sum_{k, l \in S(i, j)} c_{k l}$,

and $c_{k l}=\frac{<\Delta_{k} \cdot \Delta_{l}>-<\Delta_{k}>\cdot<\Delta_{l}>}{\Delta^{2} \sin ^{2} \theta}$

is the correlation between transverse fluctuations of spins $k$ and $l$. Now $c$ ' involves longer-range correlations than $c$, so that we can reasonably assume an upper bound to the effects of the many-atom interactions to be the case $c^{\prime}=c$; this will be taken in the following. In the ESM $\sigma^{2}$ is a constant independent of $i$ and $j$.

To fit a Hamiltonian to the interactions in equation (1.6), we imagine obtaining a configuration with given $\theta$ and $c$ from the FM state by rotating each spin an angle $\theta$ about a random axis in the $x y$ plane, so that

$\hat{\mathbf{e}}_{i}=\left(\sin \theta \cos \varphi_{i}, \sin \theta \sin \varphi_{i}, \cos \theta\right)$

with the $\phi_{i}$ correlated if necessary:

$<\hat{\mathbf{e}}_{i} \cdot \hat{\mathbf{e}}_{j}>=\cos ^{2} \theta+c_{i j} \sin ^{2} \theta$.

The couple on $\hat{\mathbf{e}}_{i}$ is

$\frac{d V}{d \theta}=-\sum_{j} 2 J_{i j} \frac{\partial}{\partial \theta_{i}}\left(\hat{\mathbf{e}}_{i} \cdot \hat{\mathbf{e}}_{j}\right)=2 J_{\mathrm{tot}} \cos \theta \sin \theta(1-c)$

where $J_{\text {tot }}$ is the sum of the exchange interactions acting on atom $i$ :

$J_{\mathrm{tot}}\left(\sigma^{2}\right)=\sum_{j} J_{i j}\left(\sigma^{2}\right)$

The energy relative to the FM state is therefore

$$
\begin{aligned}
V\left(\sigma^{2}\right)= & \int_{\sigma^{2}}^{1} J_{\text {tot }}(s) d s \\
& =V(0)-A \sigma^{2}-\frac{1}{2} B \sigma^{4} \text { if } J \text { has the form of equation (1.6) }
\end{aligned}
$$

This is equivalent to fitting the Hamiltonian to the form

$$
V=\left\lceil-A-\frac{1}{2} B \sum_{l j} \lambda_{i j} \hat{\mathbf{e}}_{i} \cdot \hat{\mathbf{e}}_{j}\right] \sum_{l j} \lambda_{i j} \hat{\mathbf{e}}_{i} \cdot \hat{\mathbf{e}}_{j}
$$

subject to the mean spherical constraint 


$$
\sum_{l}\left|\hat{\mathbf{e}}_{i}\right|^{2}=N
$$

The ESM effectively replaces the model by a spherical model with parameters that can vary with magnetisation and SRO. Below $T_{\mathrm{C}}$, the spherical model (Berlin and Kac 1952) gives a magnetisation curve

$\cos \theta(T)=\sqrt{1-\frac{T}{T_{\mathrm{C}}}}$, where $T_{\mathrm{C}}=\frac{T_{\mathrm{C}}^{\mathrm{mf}}}{G(1)}$ and $k T_{\mathrm{C}}^{\mathrm{mf}}=\frac{2}{3} J_{\mathrm{tot}}\left(\sigma^{2}\right)$,

$T_{\mathrm{C}} \mathrm{mf}$ being the mean-field Curie temperature, and a SRO parameter

$$
c=1-1 / G(1),
$$

where

$$
G(z)=\frac{1}{\Omega_{\mathrm{BZ}}} \int \frac{d^{3} \mathbf{k}}{z-\lambda(\mathbf{k})}
$$

is a lattice Green function. We then solve equations (3.5) and (3.16-7) self-consistently. We first take the $\lambda_{i j}$ as constants; these equations then reduce to a quadratic in $\cos ^{2}(\theta)$. If $B>A(1-2 c)$, the interactions are much stronger in the FM state and there is a first order transition; if $B=0$, we recover the spherical model or system $\mathrm{I}$; if $B<-A$, the FM state is unstable, $B=-A$ being system IV. This gives us the three special cases

$$
\cos \theta=\left\{\begin{array}{cc}
(1-t)^{1 / 4}, & B=A /(1-2 c) \\
(1-t)^{1 / 2}, & B=0 \\
(1-\sqrt{ } t)^{1 / 2}, & B=-A
\end{array}\right.
$$

which are plotted in figure 2 together with the magnetisation curve calculated for Fe in system II. In Fe, $J_{\text {tot }}$ decreases with magnetisation, and the ratio $J_{1} / J_{2}$ also varies; this places $\mathrm{Fe}$ in an intermediate position between the second and third cases.

Table 1 shows the calculated Curie temperatures in the systems described in $\S 2$, and table 2 shows the correlation function at $T_{\mathrm{C}}$. Values derived from the Monte Carlo simulation described in the next section are also listed. The Curie temperatures in the ESM are slightly smaller than those calculated by Monte Carlo simulation. Note that the correlation function for iron on the basis of many-atom interactions is almost identical to that of the $\mathrm{nnHm}$; this is simply because $J_{1}$ dominates in the paramagnetic state. With longer-range pairwise interactions, there is slightly more SRO. 


$\begin{array}{llll}\text { SYSTEM } & \text { MEAN FIELD } & \text { MONTE CARLO } & \text { ESM } \\ \text { I } & 5.33 J / k_{\mathrm{B}} & 3.95 \mathrm{~J} / k_{\mathrm{B}} & 3.83 \mathrm{~J} / k_{\mathrm{B}} \\ \text { II } & 2230 \mathrm{~K} & 1600 \mathrm{~K} & 1460 \mathrm{~K} \\ \text { III (1) } & 1270 \mathrm{~K} & - & 914 \mathrm{~K} \\ \text { III (2) } & 1260 \mathrm{~K} & - & 835 \mathrm{~K} \\ \text { IV } & 5.33 \mathrm{~J} / k_{\mathrm{B}} & 3.11 \mathrm{~J} / k_{\mathrm{B}} & 2.80 \mathrm{~J} / k_{\mathrm{B}}\end{array}$

Table 1. Calculated Curie temperatures for the systems described in $§ 2$. (I: nearest-neighbour Heisenberg model, II: Fe with many-atom interactions, III: Fe with longer-range pair interactions, IV extreme nonpairwise interactions.) The experimental value for $\mathrm{Fe}$ is $1040 \mathrm{~K}$.

$c(1) \quad c(2) \quad c(3) \quad c(4) \quad c(5) \quad c$

\section{Monte Carlo}

$\begin{array}{lllllll}\text { I } & 0.163 & 0.110 & 0.078 & 0.057 & 0.039 & 0.163 \\ \text { II } & 0.156 & 0.115 & 0.074 & 0.058 & 0.040 & 0.151 \\ \text { IV } & 0.172 & 0.118 & 0.084 & 0.064 & 0.044 & 0.172\end{array}$

\section{ESM}

$\begin{array}{lllllll}\text { I and IV } & 0.282 & 0.209 & 0.165 & 0.135 & 0.137 & 0.282 \\ \text { II } & 0.273 & 0.214 & 0.160 & 0.134 & 0.133 & 0.266 \\ \text { III (1) } & 0.221 & 0.250 & 0.114 & 0.131 & 0.093 & 0.280 \\ \text { III (2) } & 0.317 & 0.279 & 0.178 & 0.164 & 0.154 & 0.338\end{array}$

Table 2. Correlation function at $T_{\mathrm{C}}$ for the BCC lattice calculated by Monte Carlo simulation and the ESM for the systems described in $\$ 2$. (I: nearest-neighbour Heisenberg model, II: Fe with many-atom interactions, III: Fe with longer-range pair interactions, IV extreme non-pairwise interactions.) $c$ is the SRO parameter. 
Figure 3 compares the paramagnetic behaviour of the SRO parameter for the nnHm (I) and the extreme case $B=-A(\mathrm{IV})$. They coincide at $T_{\mathrm{C}}$; at higher temperatures the SRO is slightly larger in the extreme case than in the nnHm because of the continuing increase of $J$ with disorder.

It may be argued that the decoupling implicit in the ESM would not permit study of the type of fourspin correlations discussed in $\$ 2$ that could lead to coarse-grained disorder. For this reason we have also carried out Monte Carlo simulations of the system.

\section{Monte Carlo Simulations}

The idea behind the Monte Carlo method in statistical mechanics (see e.g. Binder and Heermann 1988) is to construct a sample of spin configurations which is representative of the canonical ensemble at a given temperature $T$. The spin on site $i$ is the unit vector

$$
\hat{\mathbf{e}}_{i}=\left(\sin \theta_{i} \cos \varphi_{i}, \sin \theta_{i} \sin \varphi_{i}, \cos \theta_{i}\right)
$$

The simulation at each temperature begins with all spins aligned ferromagnetically. At each step of the simulation a site $\mathbf{r}_{i}$ is chosen at random, and trial values of $\cos \theta_{i}$ and $\phi_{i}$ are chosen from uniform random

sequences. Separate random sequences are used for the coordinates of $\mathbf{r}_{i}, \cos \theta_{i}$ and $\phi_{i}$ to avoid possible correlations. With the Hamiltonians discussed in $\S 2$, the change in energy associated with this change is

$$
\Delta E=-2 \sum_{j}\left[\left(J_{i j} \hat{\mathbf{e}}_{i}\right)_{\text {new }}-\left(J_{i j} \hat{\mathbf{e}}_{i}\right)_{\text {old }}\right] \cdot \hat{\mathbf{e}}_{j}
$$

The $J_{i j}$ are the exchange interactions that may be dependent on order. We accept the change with probability

$$
W(\Delta E)=\left\{\begin{array}{cc}
1 & \text { if } \Delta E \leq 0 \\
e^{-\Delta E / k T} & \text { if } \Delta E \geq 0
\end{array}\right.
$$

This process is continued for as long as is required at each temperature. Averages for the required thermodynamic quantities are taken at each temperature.

The influence of the starting state is important. In the present work we start with a ferromagnetically aligned state and then allow the Monte Carlo process to reach equilibrium before averages are taken. If relaxation is slow then it may be difficult to determine how many configurations to exclude from the averages. Since we are not specifically interested in the critical region, this is not expected to be a serious problem. To a large extent the effect of the starting state on the averages can be minimised by allowing the sample to be large. In the present method we generate 1000 Monte Carlo steps per spin (MCS) after allowing a relaxation time of $200 \mathrm{MCS}$, and calculate the required thermodynamic quantities every $10 \mathrm{MCS}$, allowing $R=100$ lattice sums, labelled $v, 1 \leq v \leq R$ to be taken.

Periodic boundary conditions have been used in the simulations to approximate an infinite system. Surface effects are therefore eliminated; however, finite size effects remain. Periodic boundary conditions model the bulk behaviour fairly well for large clusters (Binder and Heermann 1988). Finite size effects 
cannot be eliminated; close to the phase transition they are important and a distinct phase transition is therefore never observed.

A Monte Carlo simulation requires knowledge of the change in energy on rotating a single moment. This could be obtained fairly directly from recursion method calculations of the moment on a rotated exchange splitting in a random medium, in the spirit of the CPA calculations of Hubbard (1979). A check at a few energies shows that such energies are consistent with the exchange interactions already calculated; we will therefore use the exchange interactions in equation (4.2) to represent the change in energy.

We should point out the danger of Monte Carlo calculations that are not based on an explicit Hamiltonian; the process must be consistent with the existence of a Hamiltonian even if we do not know what it is. The sum of the energy changes round any closed loop in phase space must be zero. We shall illustrate the problem. Suppose one has two sites $i$ and $j$, both originally spin up, in a certain environment. Then if one successively flips $i, j, i$ and $j$ in that order, it may be that $j$ is more strongly coupled to its neighbours when $i$ is up than down so that less energy is required to flip spin $j$ up than is subsequently gained by flipping it down. If spin $j$ does not have the same effect on the coupling of spin $i$ to its environment, the sum of the energy changes in this cycle will be non-zero. If detailed balance is violated in this way, the Metropolis algorithm may be more likely to go round the cycle in the direction for which the total energy change is negative. Thus we can test the consistency of the method by summing the energy changes $\Delta E$ of each accepted step. If the method is consistent, the sum will relax to an equilibrium value with small fluctuations; if it is inconsistent in the way described above, the sum will show an unbounded downwards drift. We observe here that the general trends in the Monte Carlo results are the same as in the ESM results, indicating that there is no gross discrepancy.

At every 10 MCS we calculate the following lattice sums for the reduced magnetisation $\mathbf{M}$ and the $n$th neighbour correlation $c(n)$ :

$$
\begin{aligned}
& \mathbf{M}_{v}=\frac{1}{N} \sum_{i=1}^{N} \hat{\mathbf{e}}_{i} \\
& c_{v}(n)=\left(\frac{1}{z(n) N}\right) \sum_{\substack{i j: n \text {th } \\
\text { neighbours }}} \hat{\mathbf{e}}_{i} \cdot \hat{\mathbf{e}}_{j}-\left(\mathbf{M}_{v} \cdot \mathbf{M}_{v}\right), 1 \leq n \leq 5 .
\end{aligned}
$$

The magnitude of $\mathbf{M}_{v}$ is 1 at $T=0$, and $z(n)$ is the number of $n$th neighbours.

We calculate the following Monte Carlo averages: the mean magnetisation

$$
<>=\frac{1}{R} \sum_{v=1}^{R}\left|\mathbf{M}_{v}\right|
$$

the root mean square magnetisation

$$
M_{\mathrm{rms}}=\sqrt{<M^{2}>}=\sqrt{\frac{1}{R} \sum_{v=1}^{R}\left(\mathbf{M}_{v} \cdot \mathbf{M}_{v}\right)},
$$


and the correlation function for the first 5 shells of neighbours

$$
c(n)=\frac{1}{R} \sum_{v=1}^{R} c_{v}(n) .
$$

To estimate the magnetisation per atom $M_{\infty}$ of an infinite system, we calculate $M_{\mathrm{rms}}$ for a number of system sizes $N$ from $54(3 \infty 3 \infty 3 \mathrm{BCC}$ unit cells) to $2000(10 \infty 10 \infty 10 \mathrm{BCC}$ unit cells) and fit to the asymptotic form (Paauw et al 1975)

$$
M_{\mathrm{rms}}(N)=M_{\infty}+K N^{-\frac{1}{2}}
$$

where $K$ is a constant.

Table 1 gives results for the Curie temperature for the systems described in section 2, evaluated by MFT, the Monte Carlo simulations and the ESM. The Curie temperature is estimated by eye from the magnetisation curves. In system $\mathrm{I}(\mathrm{nnHm})$ it is found to be

$$
T_{\mathrm{C}}=(3.95 \pm 0.05) J / k_{\mathrm{B}}
$$

This is to be compared with $(4.11 \pm 0.015) J / k_{\mathrm{B}}$ found by Rushbrooke et al (1974) by series expansion, $3.83 \mathrm{~J} / \mathrm{k}_{\mathrm{B}}$ from the ESM or spherical model and $5.33 \mathrm{~J} / \mathrm{k}_{\mathrm{B}}$ from MFT. It also agrees with the Monte Carlo data of Binder et al (1970). For the parameters of equation (1.7) we find $T_{\mathrm{C}}=1600 \mathrm{~K}$ for Fe, compared with an experimental value of $1040 \mathrm{~K}$. The reason why this calculation gives a larger $T_{\mathrm{C}}$ than in our previous work is that the nearest-neighbour interaction is large at the DLM end, and it is that $J$ that largely determines $T_{\mathrm{C}}$. The longer-range antiferromagnetic couplings found by Luchini and Heine (1989) do not persist in the DLM state. The table also verifies that the ESM underestimates $T_{\mathrm{C}}$ while MFT overestimates it.

Figure 4 plots the magnetisation against reduced temperature for all three systems. The magnetisation in our model of Fe falls slightly more rapidly at low temperatures, and less rapidly near $T_{\mathrm{C}}$, than in the Heisenberg system. This is because the mean exchange interaction increases with decreasing magnetisation and hence with increasing temperature, effectively stretching the temperature axis. All of our magnetisation curves deviate considerably from the experimental data; this is inevitable in a classical spin model.

The principal aim of this work is to investigate the possibility of SRO. We therefore calculate the real space correlation function $c(n)$, as defined in equations (4.5) and (4.8). Figure 5 shows the temperature dependence of the correlations for the first five shells of neighbours; table 2 lists their values at $T_{\mathrm{C}}$. It is immediately apparent that the real space correlation function in our model for Fe (II) is almost indistinguishable from that for the nearest-neighbour Heisenberg model (I). In both cases the nearestneighbour correlation, $c(1)$, is small $(0.16)$ at $T_{\mathrm{C}}$. The conclusion is therefore that we find no SRO in Fe above $T_{C} . \quad c(2)$ is slightly larger in our model II, but this is easily attributed to next-nearest-neighbour interactions rather than to non-pairwise interactions. The extreme case (IV) does show correlations decreasing with temperature rather more slowly than in the Heisenberg model; this is due to the increase of the exchange interactions with disorder.

The correlation functions have also been calculated in the ferromagnetic state in an attempt to investigate the possibility of formation of substantial clusters of reversed spin. The results, when compared 
with the Heisenberg model, do not support this claim. The difference between our data and the Heisenberg model reflect the effective stretching of the temperature scale and the change in the range of interactions with temperature. If substantially sized clusters did form then the nearest and next nearest neighbour correlation functions in the ferromagnetic state would be much larger than we find. These would also imply a higher degree of SRO.

We can also check the form of the interactions against the temperature-dependence of the spin wave dispersion in Fe. Magnon-magnon interactions lead to a decrease of magnon energies with temperature in the Heisenberg model. Lynn (1975) reported a smaller renormalisation, with magnon energies only weakly dependent on temperature (even above $T_{\mathrm{C}}$ ). This observation, at least within the ferromagnetic phase, could

be consistent with the increase in the $J_{i j}$ with disorder. This increase would compensate for the normal energy renormalisation; calculations are currently in progress.

\section{Conclusions}

No evidence has been found in our calculations that Fe behaves in a substantially different way from the nearest-neighbour Heisenberg model. We use an effective classical spin Hamiltonian extracted from electronic structure calculations, a Hamiltonian which may differ from the nnHm in two important ways. The interactions may be of long range and oscillate in sign. Such interactions can yield substantial SRO (Shastry et al 1981) but are not supported by the electronic calculations. The effect of disorder and finite temperature would reduce the amplitude of the longer-range interactions, making this mechanism unlikely. The other approach, the one discussed here based on the results of LH, is to assume substantial many-atom interactions. While many-atom interactions could lead to coarse-grained disorder, they would also strongly distort the magnetisation curves in the ferromagnetic phase.

The question is: if one could calculate the full partition function in the static approximation, given a suitable parametrisation of the band structure of iron, would the SRO differ substantially from that of a nearest-neighbour Heisenberg model? It would require an unfeasible amount of CPU time to derive a Hamiltonian for arbitrary configurations of the magnetisation and to calculate the resulting partition function. We cannot say what would result from such a study. We have however taken a reasonably large sample of configuration space. Our other approximation is the static approximation, which is roughly the magnetic equivalent of the Born-Oppenheimer approximation (You and Heine, 1982). The magnetisation is taken as a classical field precessing slowly in comparison with electron hopping, and the energy of electrons moving on such a frozen configuration is calculated. Although the spin wave stiffness can be calculated successfully from electronic structure data (Luchini and Heine 1989), the physics of the entry of the spin waves into the Stoner continuum at $q_{\mathrm{c}}$ is missing from our static Hamiltonian. The answer to our question that we have now come to is that we cannot account for the apparent SRO indicated by paramagnetic neutron scattering in this approximation. The observed correlation function may result from dynamic or quantum effects, important for wave vectors above $q_{\mathrm{c}}$ but omitted in the static approximation, which does not include the dynamics. Such effects, if they can be expressed as a classical Hamiltonian, would have to lead to effective exchange interactions of a substantially different form from those obtained in the static approximation if the observed correlation functions are to be explained. The conclusion however is that electronic structure calculations on static configurations cannot on their own explain the correlation functions observed by neutron scattering.

\section{Acknowledgments}

We are very grateful to Kurt Ziebeck for discussions on the interpretation of the neutron data, and also wish to thank the SERC (KSC) and the Schweizer Nationalfonds and the Fondazione Angelo della Riccia, Florence (MUL) for financial support. 


\section{References}

Berlin T H and Kac M 1952 Phys Rev 86 821-35

Binder K and Heermann D W 1988 Monte Carlo Simulation in Statistical Physics An Introduction (Berlin: Springer)

Binder K, Rauch H and Wildpaner V 1970 J. Phys. Chem. Solids 31 391-7

Brown H A 1971 Phys. Rev. B4 115-21

Brown P J, Capellmann H, Déportes J, Givord D and Ziebeck K R A 1982 J. Magn. Magn. Mater. 30 243-8

Edwards D M 1980 J. Magn. Magn. Mater. 15-18 262-8

1983 J. Magn. Magn. Mater. 36 213-6

Haines E M, Clauberg R and Feder R 1985 Phys. Rev. Lett. 54 932-4

Hasegawa H 1980 J. Phys. Soc. Japan 49 178-88, 963-71

Haydock R 1980 Solid State Phys. 35 215-94

Heine V and Joynt R 1988 Europhys. Lett. 5 81-5

Heine V, Liechtenstein A I and Mryasov O N 1990 Europhys. Lett. 12 545-50

Heine V, Samson J H and Nex C M M 1981 J. Phys. F: Met. Phys. 11 2645-62

Holden A J and You M V 1982 J. Phys. F: Met. Phys. 12 195-214

Hubbard J 1979 Phys. Rev. B19 2626-2636, B20 4584-4595

Johnson S M, Chaumet M, Neumann K-U, Thoma R and Ziebeck K R A 1987 J. Magn. Magn. Mater. 67 295-310

Kisker E, Clauberg R and Gudat W 1985 Z. Phys. B61 453-6

Korenman V, Murray J L and Prange R E 1977 Phys. Rev. B16 4032-47, 4048-57, 4058-62

Landolt-Börnstein 1962 Zahlenwerte und Funktionen II 9 p 1.17 (Berlin: Springer)

Lin-Chung P J and Holden A J 1981 Phys. Rev.B23 3414-20

Lipiński S 1989 Z. Phys. B74 241-6

Lowde R D, Moon R M, Pagonis B, Perry C H, Sokoloff J B, Vaughan-Watkins R S, Wiltshire M C K and Crangle J 1983 J. Phys. F: Met. Phys. 13 249-79

Luchini M U and Heine V 1989 J. Phys.: Condens. Matter 1 8961-77

1991a Europhys. Lett. 14 609-12

$1991 \mathrm{~b}$ to be published

Lynn J W 1975 Phys. Rev. B11 2624-37

Mook H A, Lynn J W and Nicklow R M 1973 Phys. Rev. Lett. 30 556-9

Oguchi T, Terakura K and Hamada N 1983 J. Phys. F: Met. Phys. 13 145-60

Paauw Th T A, Compagner A and Bedeaux D 1975 Physica 79A 1-17

Rushbrooke G S, Baker G A Jr and Wood P J 1974 in Phase Transitions and Critical Phenomena vol 3, ed Domb C and Green M S, Academic (London) 245-356

Samson J H 1983 Phys. Rev.B28 6387-93 1989 J. Phys.: Condens. Matter $16717-29$

Shastry B S, Edwards D M and Young A P 1981 J. Phys. C: Solid State Phys. 14 L665-70

Shirane G 1984 J. Magn. Magn. Mater. 45 33-37

Shirane G, Böni P and Wicksted J P 1986 Phys. Rev. B33 1881-5

Small L M and Heine V 1984 J. Phys. F: Met. Phys. 14 3041-52

You M V and Heine V 1982 J. Phys. F: Met. Phys. 12 177-94 


\section{Figure captions}

1. Nearest- and next-nearest-neighbour exchange interactions as functions of the mean square magnetisation $\sigma^{2}$ in the surrounding shell.

2. $\quad$ Magnetisation in the ESM for many-atom interactions given in equation (3.18). The curves from top to bottom are (i) $B=A /(1-2 c)$ (interactions strongest in FM state), (ii) $B=0$ (Heisenberg model), (iii, bold line) parameters for $\mathrm{Fe}$ and (iv) $B=-A$ (interactions vanishing in FM state).

3. Nearest-neighbour correlation above $T_{\mathrm{C}}$. Bold line: extreme case $B=-A$; thin line: Heisenberg model.

4. Monte Carlo simulations of magnetisation. Thin line: system I (Heisenberg model); Bold line: system II (Fe); dotted line: system IV (extreme case). Crosses: experimental data for Fe (LandoltBörnstein 1962).

5. Monte Carlo simulations of first to fifth neighbour correlations (from top to bottom) for the Heisenberg model (lower thin lines), $\mathrm{Fe}$ (bold lines) and extreme case (upper thin lines). 


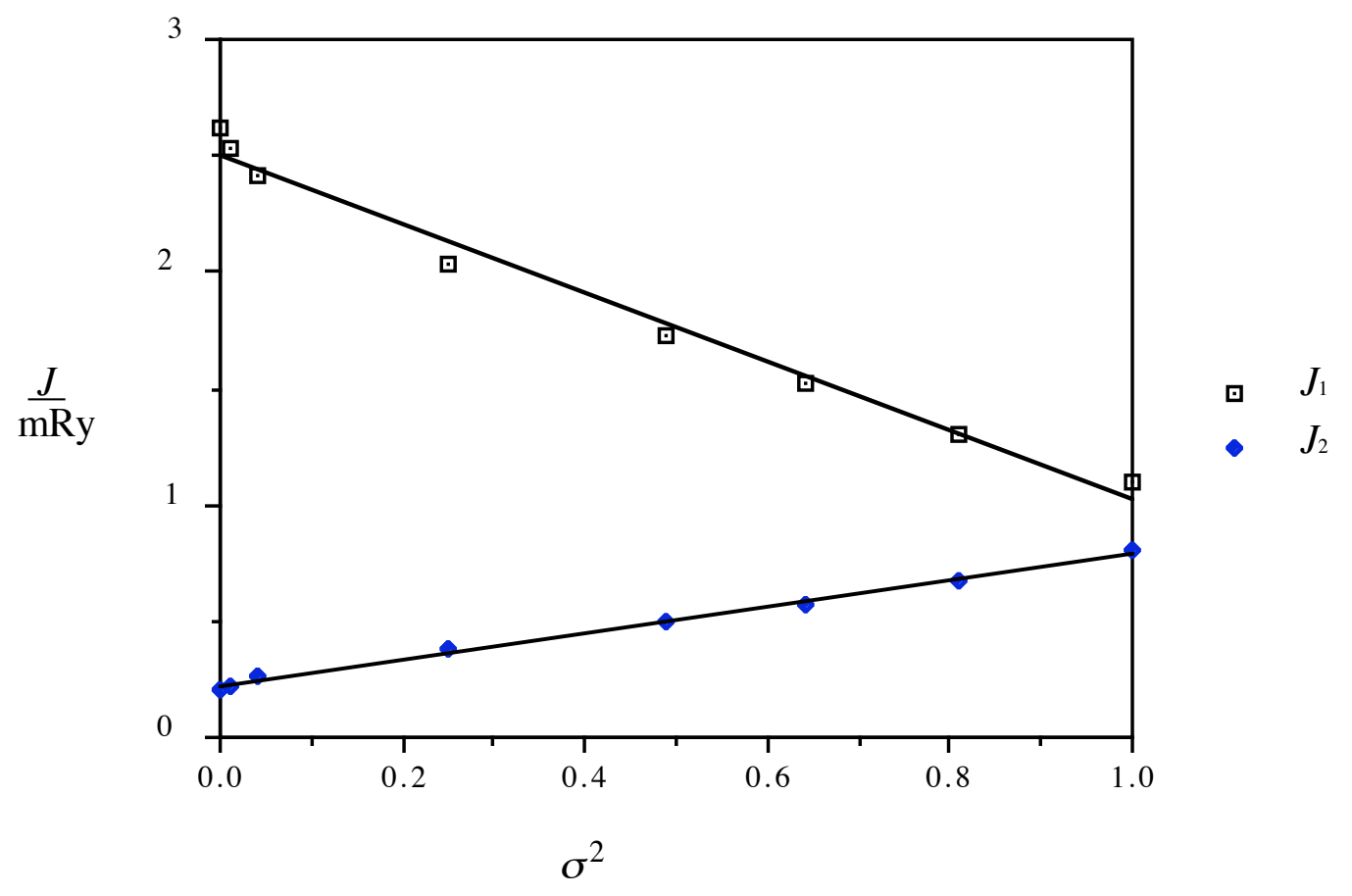

Figure 1 


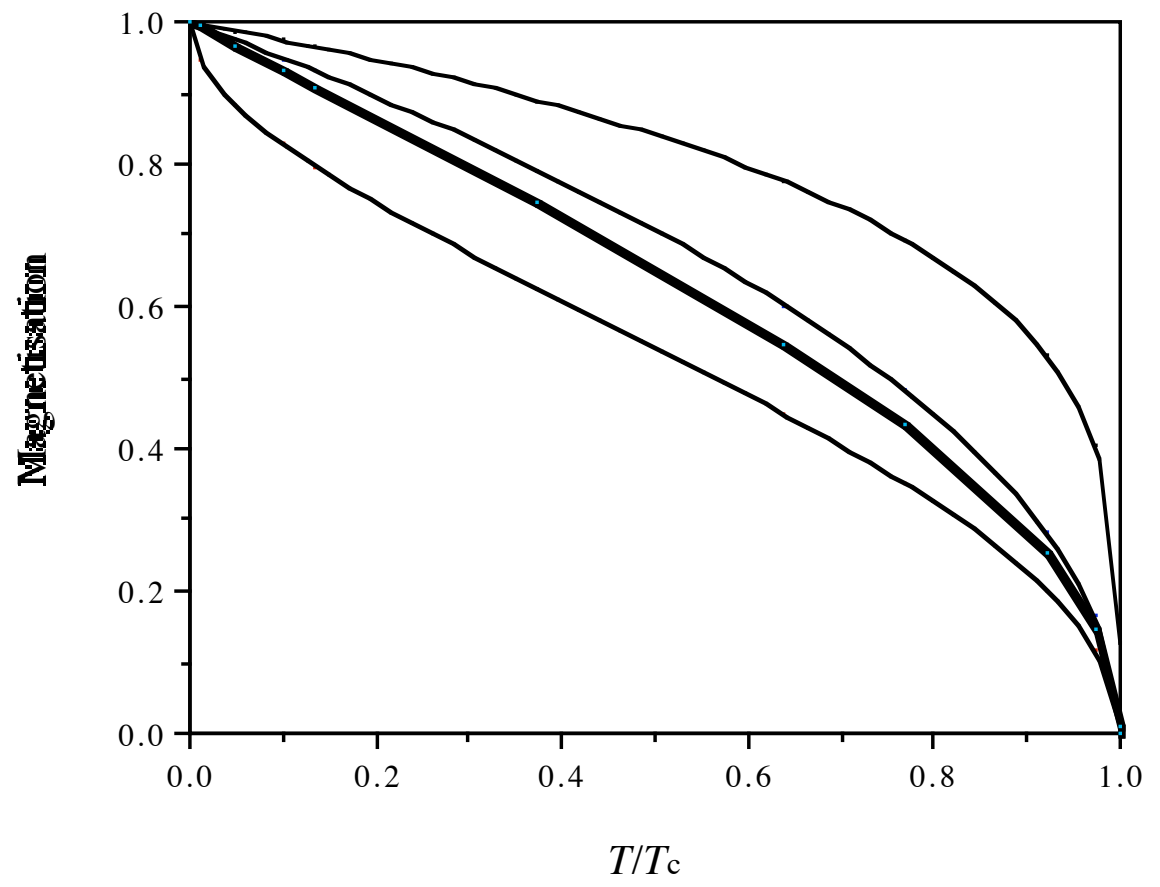

Figure 2 


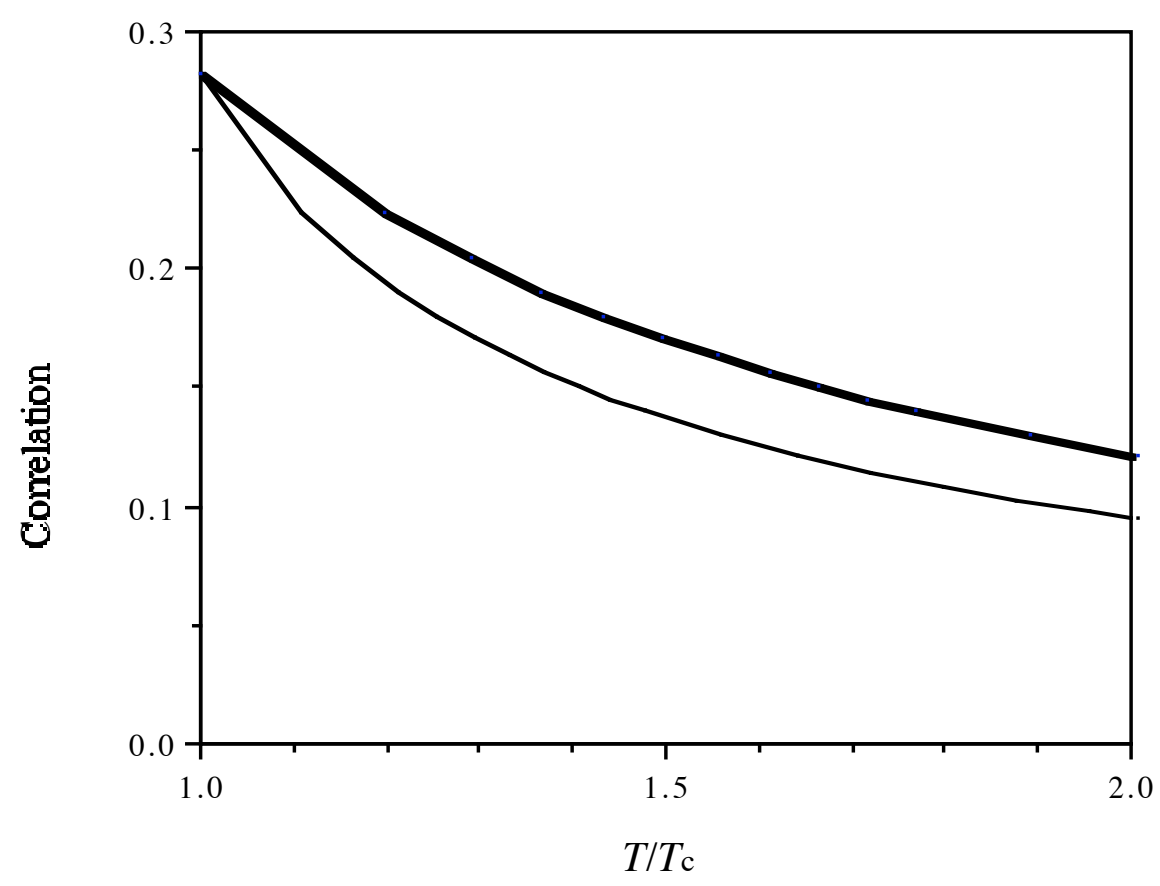

Figure 3 


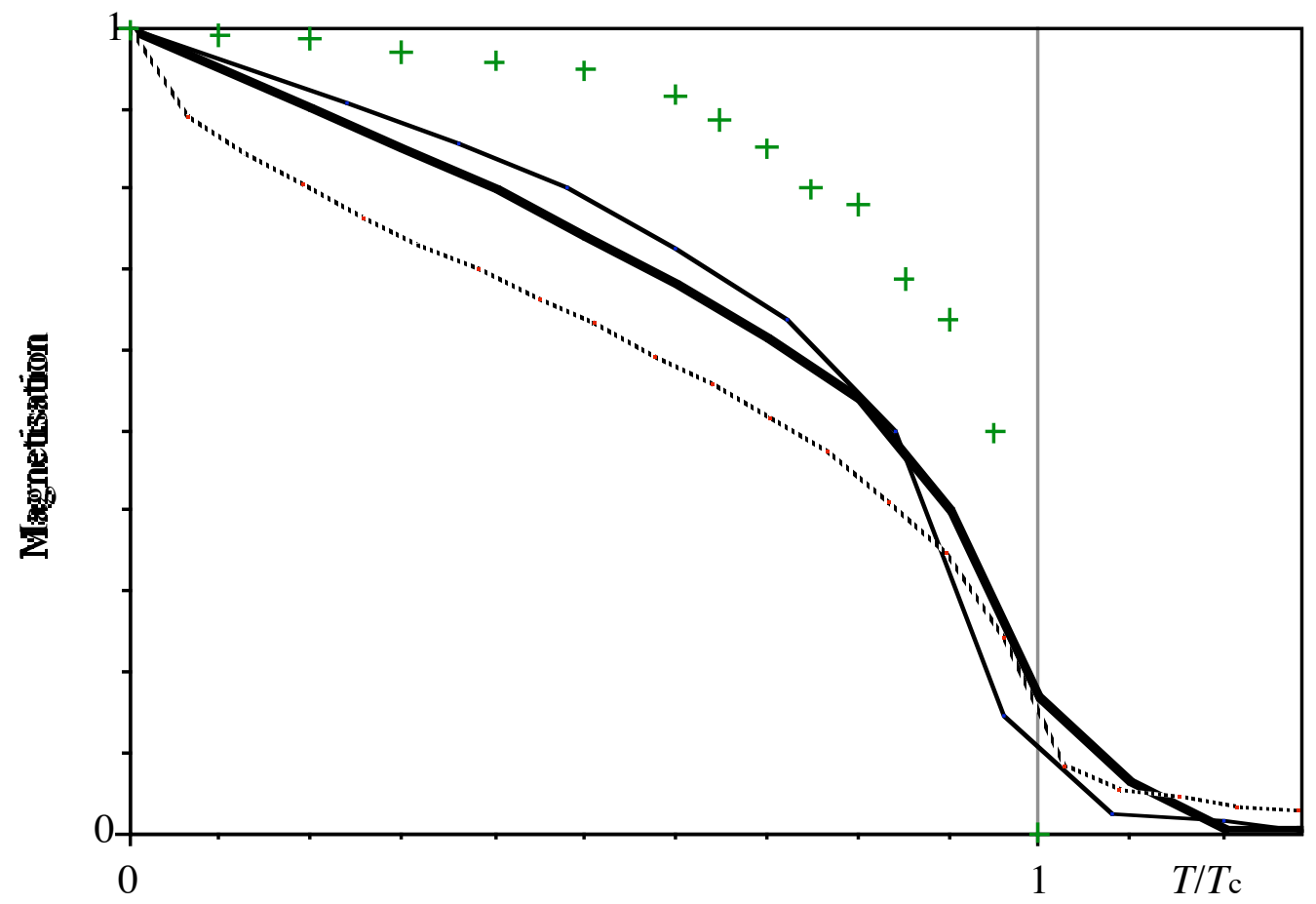

Figure 4 


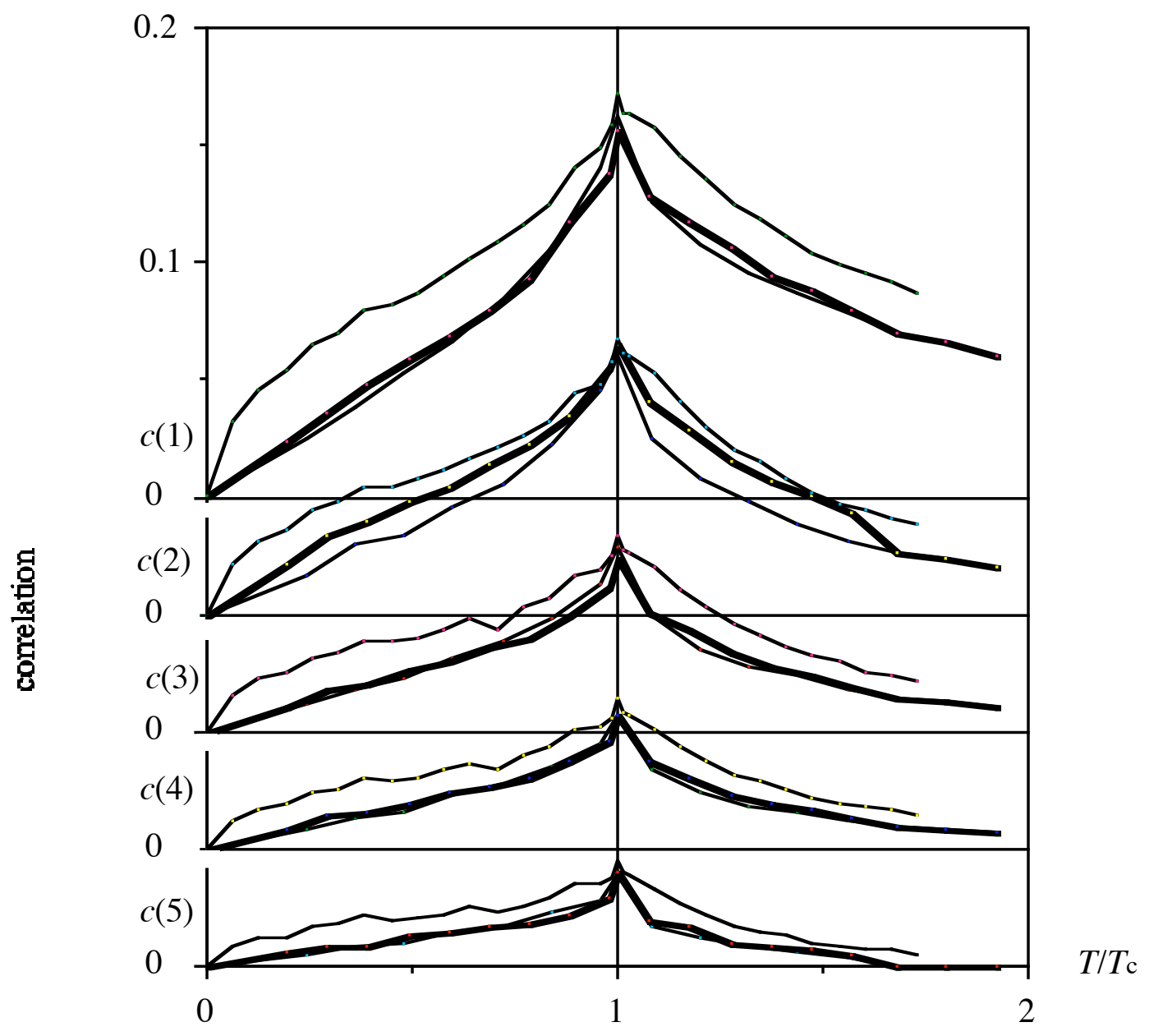

Figure 5 